\title{
A Novel Porous Media Permeability Model Based on Fractal Theory and Ideal Particle Pore-Space Geometry Assumption
}

\author{
Yongquan $\mathrm{Hu}^{1}{ }^{1}$, Qiang Wang ${ }^{1, *}$, Jinzhou Zhao ${ }^{1} \mathbb{D}$, Shouchang $\mathrm{Xie}^{2}$ and Hong Jiang ${ }^{2}$ \\ 1 State Key Laboratory of Oil-Gas Reservoir Geology \& Exploitation, Southwest Petroleum University, \\ Chengdu 610500, China; 201621000663@stu.swpu.edu.cn (Y.H.); 201811000142@stu.swpu.edu.cn (J.Z.) \\ 2 Xinjiang Oilfield Company Development Company, Karamay 834000, China; \\ xieshouc@prtrochina.com.cn (S.X.); hongj@prtrochina.com.cn (H.J.) \\ * Correspondence: 201822000147@stu.swpu.edu.cn
}

Received: 28 October 2019; Accepted: 17 January 2020; Published: 21 January 2020

\begin{abstract}
In this paper, a novel porous media permeability model is established by using particle model, capillary bundle model and fractal theory. The three-dimensional irregular spatial characteristics composed of two ideal particles are considered in the model. Compared with previous models, the results of our model are closer to the experimental data. The results show that the tortuosity fractal dimension is negatively correlated with porosity, while the pore area fractal dimension is positively correlated with porosity; The permeability is negatively correlated with the tortuosity fractal dimension and positively correlated with the integral fractal dimension of pore surface and particle radius. When the tortuosity fractal dimension is close to 1 and the pore area fractal dimension is close to 2, the faster the permeability changes, the greater the impact. Different particle arrangement has great influence on porous media permeability. When the porosity is close to 0 and close to 1 , the greater the difference coefficient is, the more the permeability of different arrangement is affected. In addition, the larger the particle radius is, the greater the permeability difference coefficient will be, and the greater the permeability difference will be for different particle arrangements. With the increase of fractal dimension, the permeability difference coefficient first decreases and then increases. When the pore area fractal dimension approaches 2 , the permeability difference coefficient changes faster and reaches the minimum value, and when the tortuosity fractal dimension approaches 1 , the permeability difference coefficient changes faster and reaches the minimum value. Our research is helpful to further understand the connotation of medium transmission in porous media.
\end{abstract}

Keywords: porous media; fractal theory; particles model; permeability; tube bundle model

\section{Introduction}

Fibrous and reservoir rocks are porous media with complex microstructure. It is very important to reasonably characterize the pore structure and predict the permeability of porous media for industrial application and petroleum exploration and development [1-3]. Pore structure plays an important role in the properties of porous media. However, due to the complexity of microstructure and irregularity of pore structure, it is always a challenging task to predict permeability [4-8]. The microstructures of oolitic graintone and dolograinstone can be found in previous study $[9,10]$. There are plenty of rounded particles that make up the skeleton of the rock and the blue areas represent random pores or micropores between particles. These pores are randomly distributed in space, with sizes spanning several orders of magnitude and connecting with each other through thick channels, forming a complex network of pores. In order to achieve qualitative research, many researchers use this particle model (see Figure 1a) 
to construct rock space, with the purpose of reconstructing the complex pore structure of the rock, so as to more accurately describe the properties of the rock [11]. Gebart [12] regards the cross section of fibrous porous media as the cross section of circles of equal diameter arranged in a fixed geometry (I and II in Figure 1a), and deduces the permeability model of the fluid flowing along the fiber and perpendicular to the fiber direction. The model establishes the relationship between the permeability and particle size and the volume fraction of the fiber. Since the randomness and complexity of pore space distribution are not considered in this model, the permeability of porous media with low porosity predicted by this model is quite different from the experimental results [13]. Therefore, fractal theory was introduced to study the relationship between pore structure and permeability of porous media from a more realistic perspective.

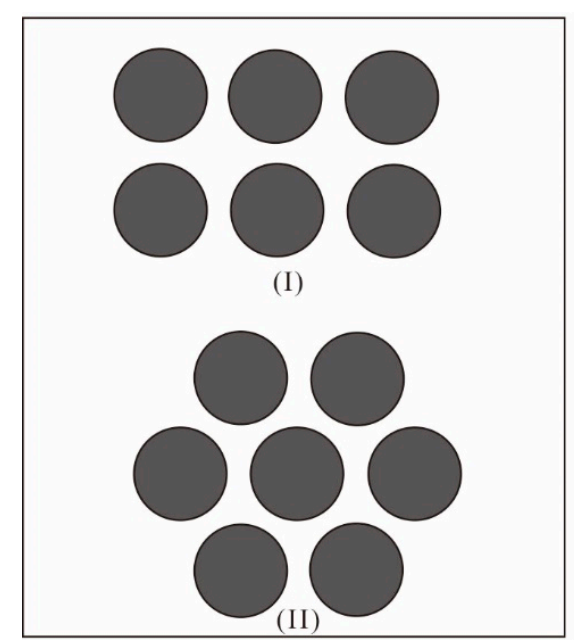

(a) 2D plane model

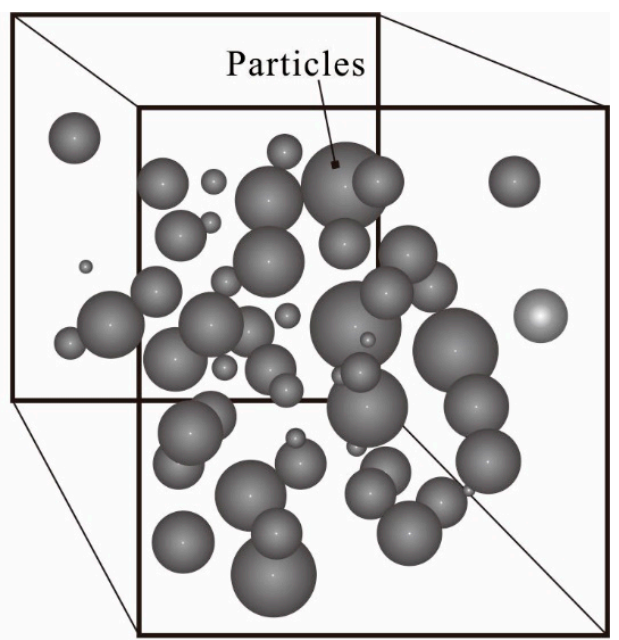

(b) Porous media

Figure 1. Schematic diagram of porous media model.

Fractal theory describes a natural phenomenon, which allows the self-similarity of objects to represent the properties of objects or scale invariance, and is an effective method to describe the complexity and irregularity of pores in porous media and their related macroscopic transport characteristics [14-17]. The application of fractal theory to the study of porous media is actually to use an appropriate method or model to characterize the structural characteristics of porous media, and then analyze its properties such as transmission and strain $[18,19]$. At present, there have been many research achievements that use fractal theory and technology to characterize the structure of porous media and analyze its permeability. According to the characterization models of porous media, there are mainly permeability models based on fractal capillary bundle model, fractal improvement for the limitations of the classical Kozeny-Carman (KC) permeability equation, random and deterministic mass fractal porous media permeability model, and fractal effective permeability models based on the Bautista Manero Puig (BMP) model [20-23]. Based on the fractal characteristics of fibrous porous media, Yu et al. [24] used the fractal capillary bundle model to put forward the fractal plane permeability model applicable to various fibrous media earlier. Studies have shown that fiber preforms mainly depends on the fiber bundles of macro pore permeability. Based on Yu's research, $\mathrm{Xu}$ and $\mathrm{Yu}$ [25] introduced the cross-sectional area of the unit and developed a fractal permeability model for homogeneous porous media. The model is not limited to fiber materials, but also applicable to other fractal porous media. Combining the maximum hydraulic diameter with filament diameter, Xiao et al. [26] expressed the maximum pore diameter as the relationship between porosity and particle size, fractal dimension, and further obtained the dimensionless permeability expression of porous fiber gas diffusion layer. The original fractal permeability model is based on the fractal power law distribution of pore size and Hagen-Poiseuille (H-P) flow equation of circular curved capillary. $\mathrm{Xu}$ and $\mathrm{Yu}$, and Xiao et al.'s 
research is based on the same conditions and assumptions [25-28]. Their models can well fit the permeability test results of the existing high-porosity fiber materials, but the permeability of the low-porosity porous media is not well fitted. Considering the discreteness and discontinuity of fractal geometry, Shou et al. [29] proposed a differential permeability model of fiber porous media, which can fit the permeability results of fiber porous media with a wider porosity range. According to the theory of fluid mechanics, H-P flow is dominant in pore media only when the Knudsen number is less than 0.01. When the Knudsen number is greater than 10, the Knudsen number is dominant. Considering these factors, Zhang et al. developed a new fractal gas permeability model for porous fiber films [30]. Considering electro kinetic phenomena, Zhu et al. studied the flow behavior of porous fibrous media using fractal technique, and derived a fractal permeability model [31]. Using similar methods and theories, Zhu et al. also studied the heat and mass transfer characteristics of fibrous porous media and took capillary force into consideration. However, none of the above fractal studies considered the influence of this factor [32]. Costa, Othman et al. established the porosity permeability model based on the fractal hypothesis of porous media particles and pore area, improved the classic Kozeny Carman permeability equation, and re-verified its validity $[13,33]$. Considering the porosity connectivity probability, Cihan et al. developed a three-dimensional solid mass fractal porous media permeability model [34]. According to the classic Sierpinski carpet quality fractal model [35-38], Pia and Sanna proposed a new combination of structural units, formed a intermingled fractal units model (IFU), and studied the transmission characteristics of porous media such as permeability and thermal conductivity [39-41]. Subsequent researchers have proposed new pore media models through this method. Similar to the above fractal study on permeability of fibrous porous media, on the basis of Yu's study [24], Turcio et al. studied the effective permeability of non-Newtonian by using the fractal capillary bundle model, and calculated the effective permeability by using the Bautiista Manero Puig (BMP) model [42,43].

With the development and application of fractal theory, more and more factors are considered into the permeability model, and the fit between model results and experimental results is getting higher and higher. However, in researches on porous media permeability based on particle model, most permeability models are still based on two-dimensional particle model, which cannot fully reflect the three-dimensional influence of pore geometry space [44]. In addition, most of the modified KC equations are more applicable to porous media with large porosity, while there are few research results on porous media with small porosity [45-47]. In this paper, the pore space is considered to be randomly distributed and its size spans over two orders of magnitude, which satisfies the scaling law of fractals. In this paper, the matrix structure of porous media is composed of spherical particles (as show in Figure $1 b$ ), and then the three-dimensional irregular pore space composed of equal-diameter particles according to ideal geometric model is approximately transformed into capillary bundle model. Finally, the relationship between permeability and pore structure parameters, pore area fractal dimension and capillary tortuosity fractal dimension is established by using fractal principle. In addition, the permeability models of the two particle combination modes were deduced, and finally the permeability models of the loose mode and the compact mode were obtained. Compared with the experimental data and the results of existing analytical formulas, our model is reliable and accurate.

\section{Mathematical Model}

\subsection{Fractal Characteristics of Spherical Particles Matrix}

In this paper, the matrix is assumed to be composed of spherical particle clusters, each cluster is composed of particles with the same radius, and the matrix particle radius between clusters is randomly distributed, so there are pores of different sizes in the porous media, and these pore Spaces satisfy the fractal scale theorem $[13,48]$. As shown in Figure 2, there are two types of clusters: loose mode I cluster (Figure 2a) and compact mode II cluster (Figure 2d). Type I cluster is the structural combination of particles that can form the largest pore space, while type II cluster is the structural 
combination of particles that can form the smallest pore space. Type I clusters (Figure 2a) consist of eight spherical particles forming a matrix particle cluster. The central point connecting each particle can form a cube. Cutting along the cube surface can form a matrix cube unit as shown in Figure $2 b$. From Figure $2 b, c$, eight one eighth of the matrix particles constitute an irregular matrix pore space, which can maximize the matrix pore space. Type II clusters (Figure 2d) consist of four matrix particles, which can form rhombohedrons by connecting the central points of the particles. Cutting along the surface of the rhombohedron can form a unit as shown in Figure 2e,f.

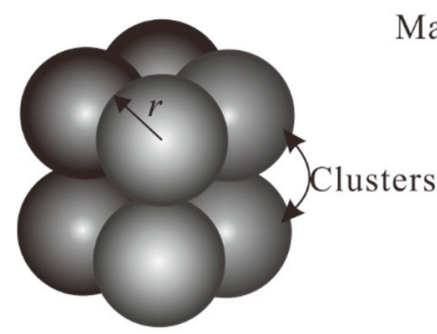

(a)

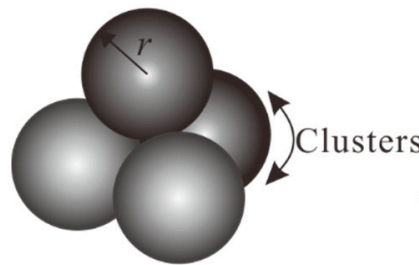

(d)

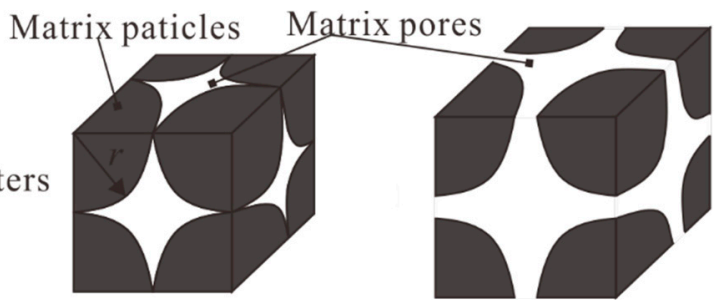

(b)

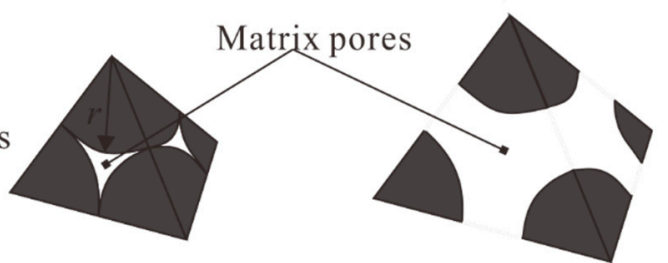

(e) (f)

Figure 2. Schematic diagram of structural model of ideal pore space. (a) Arrangement of loose particles;

(b) Ideal loose arrangement; (c) Conventional loose arrangement; (d) Compact particles arrangement;

(e) Ideal most compact arrangement; (f) Regular compact permutation.

For matrix units composed of type I clusters according to ideal geometry, they are mainly composed of matrix particles and pore space, as shown in Figure 3a. The porosity can be expressed as the relationship between cube volume and matrix particle volume [13]:

$$
\varphi=\frac{V_{\mathrm{c}}-\frac{4}{3} \pi r^{3}}{V_{\mathrm{c}}}
$$

where $\varphi$ is the effective reservoir porosity; $V_{\mathrm{c}}$ represents the volume of the cluster cube; $r$ is the radius of the matrix particle.

Through Equation (1), the cubic unit volume can be deduced as follows:

$$
V_{\mathrm{c}}=\frac{4 \pi r^{3}}{3(1-\varphi)}
$$

It can be seen from Equation (2) that the volume of cubic unit is related to particle radius and porosity. Since $V_{\mathrm{c}}$ represents the cube space formed by cluster matrix particles, there is:

$$
V_{\mathrm{c}}=L_{0}^{3}
$$

where $l_{0}$ is the side length of a matrix cubic unit. 


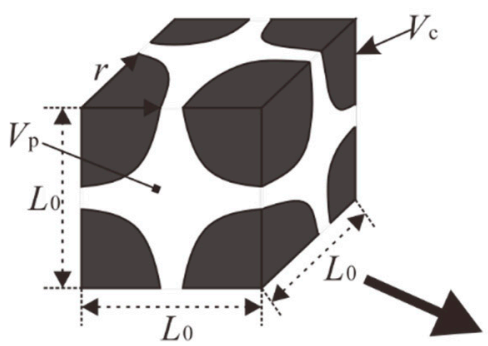

(a)

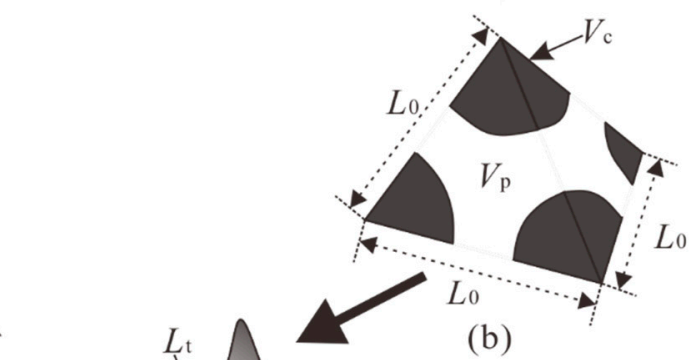

(b)
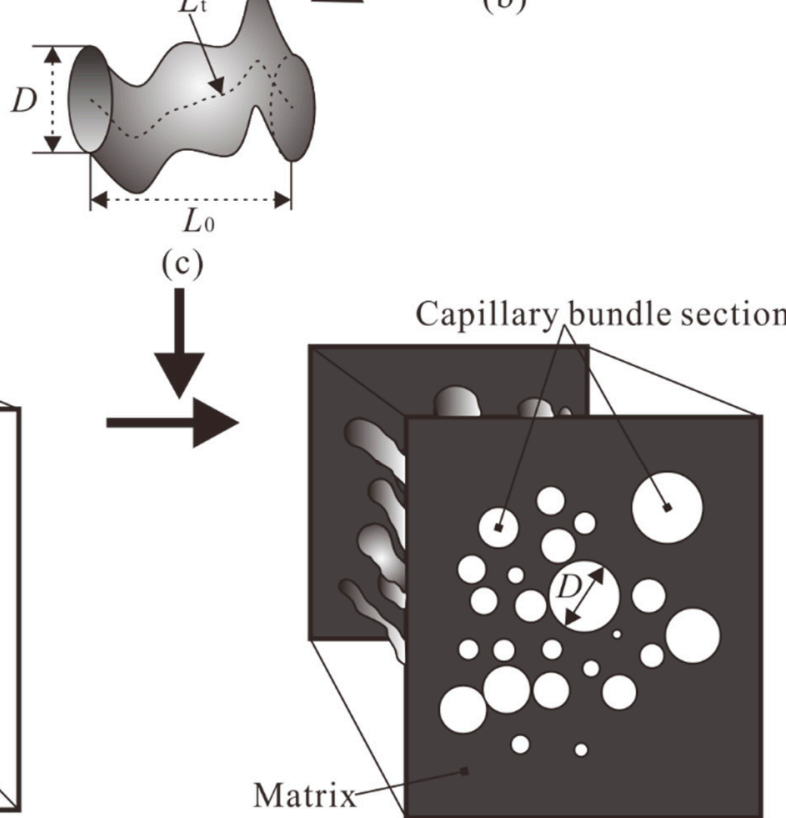

(d)

(e)

Figure 3. Schematic diagram of irregular pore space transformed into capillary bundle model. (a) conventional loose arrangement; (b) regular compact permutation; (c) capillary channel; (d) actual particle model; (e) transformed capillary model.

Combining Equations (2) and (3), the expression can be obtained:

$$
L_{0}=r \times \sqrt[3]{\frac{4 \pi}{3(1-\varphi)}}
$$

The maximum matrix pore volume $V_{\mathrm{p}, \max }$ can be expressed as:

$$
V_{\mathrm{p}, \max }=V_{\mathrm{c}}-\frac{4 \pi r^{3}}{3}=\frac{4 \pi r^{3}}{3} \frac{\varphi}{1-\varphi}
$$

When the fluid flows through the matrix element, it mainly passes through the three sections shown in Figure 4, which not only passes through the maximum section of the ideal geometric square pore in the middle, but also passes through the minimum section of the ideal geometric irregularity on both sides [13]. The rationality of pore space is considered. Therefore, the irregular matrix pore space composed of solid particles is approximately equivalent to capillary bundle, and the capillary diameter on the equivalent cross section satisfies the fractal scale law, then:

$$
V_{\mathrm{p}}=L_{\mathrm{t}} \frac{\pi D^{2}}{4}
$$


where $D$ is the diameter of the capillary bundle section; $L_{t}$ is the actual length of tortuous bundle, and the ratio of its value to the characteristic length of the external surface can be expressed as:

$$
\tau=\frac{L_{t}}{L_{0}}
$$

Formula (7) is the traditional definition of tortuosity, whose value can be taken as average tortuosity. Yu and Li, Yun et al. and Kou et al. studied the tortuousness model of two-dimensional, three-dimensional porous media and mixed porous media composed of square and circular particles [49-52]. A series of relationships between tortuosity and porosity were established.

Combined with Equations (4)-(7), the maximum tortuous capillary bundle cross-section diameter obtained by cubic element approximation can be derived:

$$
D_{\max }=4 \sqrt[3]{\frac{1}{9}} \sqrt[6]{\frac{1}{4 \pi}} \sqrt{\frac{\varphi}{\tau}} \sqrt[3]{\frac{1}{(1-\varphi)}} r
$$

For type II clusters, the porosity in this structure can also be expressed as the relationship between rhombohedral volume and matrix particle volume:

$$
\varphi_{\mathrm{e}}=\frac{V_{\mathrm{c}}-\frac{2}{9} \pi r^{3}}{V_{\mathrm{c}}}
$$

The pore volume expressed in terms of effective porosity, cementing ratio and particle radius can be obtained from Equation (9):

$$
V_{\mathrm{c}}=\frac{2 \pi r^{3}}{9(1-\varphi)}
$$

Rhombohedron is a regular tetrahedron. According to its geometric characteristics, its volume can be expressed as:

$$
V_{\mathrm{c}}=\frac{\sqrt{2}}{12} L_{0}^{3}
$$

Side length of rhombohedron can be obtained by combining Equations (11) and (10):

$$
L_{0}=r \times \sqrt[3]{\frac{24 \pi}{9 \sqrt{2}(1-\varphi)}}
$$

According to Equation (10), the maximum matrix pore volume of rhombohedron with porosity of $\varphi$ can be obtained as:

$$
V_{\mathrm{p}, \max }=V_{\mathrm{c}}-\frac{2 \pi r^{3}}{9}=\frac{2 \pi r^{3}}{9} \frac{\varphi}{1-\varphi}
$$

By combining Equations (6), (7), (12) and (13), the maximum sectional diameter of tortuous bundle approximately obtained by rhombohedron can be obtained as follows:

$$
D_{\max }=\frac{2 \sqrt{2}}{3} \sqrt[6]{\frac{9 \sqrt{2}}{24 \pi}} \sqrt{\frac{\varphi}{\tau}} \sqrt[3]{\frac{1}{(1-\varphi)}} r
$$

It can be seen from Equations (8) and (14) that the two equations have the same form, and the capillary diameter is a function of particle radius, porosity and tortuosity. According to $\mathrm{Yu}$ and $\mathrm{Li}$ [49], tortuosity is also a function of porosity. Therefore, the key parameters to determine the equivalent capillary diameter are particle radius and porosity. 


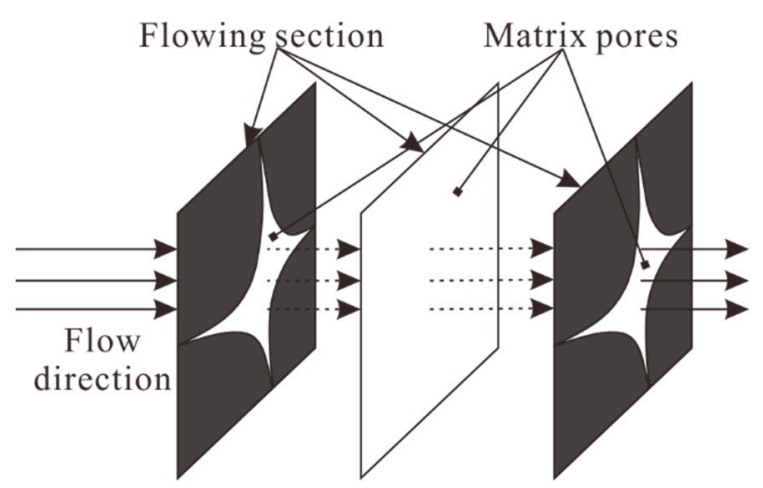

Figure 4. Fluid flow through the pore section diagram.

\subsection{Fractal Capillary Bundle Model for Porous Media}

The capillary bundle model is often used to simulate the flow and transport characteristics of porous media. The pore size distribution and the fractal scale relationship of curved streamline constitute the basis of fractal capillary bundle model. Most natural rocks have fractal characteristics in a certain range. For example, sandstone, shale and carbonate rocks are self-similar in a range of 3 to 4 orders of magnitude. Fractal dimension can be used to quantitatively describe the characteristics of pore size distribution [53]. In fractal porous media, the cumulative number of pores with diameters larger than the scale $N$ follows the scaling law relationship:

$$
N(\geq D)=\left(\frac{D_{\max }}{D}\right)^{D_{\mathrm{p}}}
$$

where $D_{\mathrm{p}}$ is pore area fractal dimension. When the value is $0<D_{\mathrm{p}}<2$, it denotes two-dimensional space, and when $0<D_{\mathrm{p}}<3$, it denotes three-dimensional space. According to Yu's research, the fractal dimension of pore area can be expressed as [24,49]:

$$
D_{\mathrm{p}}=d-\frac{\ln \varphi}{\ln \frac{D_{\min }}{D_{\max }}}
$$

where $D_{\min }$ is the smallest diameter of capillary tube. $d$ is equal to 2 (two-dimension) or 3 (three-dimension).

If the pore diameter ranges from $D_{\min }$ to $D_{\max }$, the total number of medium pores under the condition $D>D_{\min }$ can be obtained. So we know from Equation (15):

$$
\mathrm{N}_{\mathrm{p}}\left(D>D_{\min }\right)=\left(\frac{D_{\max }}{D_{\min }}\right)^{D_{\mathrm{p}}}
$$

Differentiating Equation (15), we can get:

$$
-\mathrm{d} N=D_{\mathrm{p}} D_{\max }^{D_{\mathrm{p}}} D^{-\left(D_{\mathrm{p}}+1\right)} \mathrm{d} D
$$

Equation (18) gives the number of pores in the interval $D$ and $D+\mathrm{d} D$, and $-\mathrm{d} N>0$ indicates that the number of pores decreases with the increase of pore diameter. Divide Equations (17) and (18) to get:

$$
-\frac{\mathrm{d} N}{N_{\mathrm{p}}}=D_{\mathrm{p}} D_{\min }^{D_{\mathrm{p}}} D^{-\left(D_{\mathrm{p}}+1\right)} \mathrm{d} D=f(D) \mathrm{d} D
$$

where $f(D)=D_{\mathrm{p}} D_{\min }^{D_{\mathrm{p}}} D^{-\left(D_{\mathrm{p}}+1\right)}$ is the probability density function of pore distribution, which should meet the normalization condition: 


$$
\int_{-\infty}^{+\infty} f(D) \mathrm{d} D=\int_{D_{\min }}^{D_{\max }} f(D) \mathrm{d} D=1-\left(\frac{D_{\min }}{D_{\max }}\right)^{D_{\mathrm{p}}}=1
$$

The condition for Equation (20) to be true must be:

$$
\left(\frac{D_{\min }}{D_{\max }}\right)^{D_{\mathrm{p}}}=0
$$

Curved capillary or curved streamline of fluid flow also has fractal characteristics. Tortuosity depends on measurement scale and fractal dimension of streamline, which can better reflect the characteristics of curved capillary streamline. The tortuosity fractal dimension is considered to be a more fundamental parameter than permeability. The scaling relationship between the length of curved streamline and the characteristic length of the medium in porous media can be expressed as [48,54]:

$$
\begin{gathered}
L_{\mathrm{t}}(D)=L_{0}^{D_{\mathrm{t}}} D^{1-D_{\mathrm{t}}} \\
D_{\mathrm{t}}=1+\frac{\ln \tau_{\mathrm{av}}}{\ln \frac{L_{0}}{D_{\mathrm{av}}}}
\end{gathered}
$$

where $D_{\mathrm{t}}$ is the tortuosity fractal dimension. Average capillary diameter $D_{\mathrm{av}}$ and average tortuosity $\tau_{\text {av }}$ can be expressed as [49,55]:

$$
\begin{gathered}
D_{\mathrm{av}}=\int_{D_{\min }}^{D_{\max }} D f(D) \mathrm{d} D=\frac{D_{\mathrm{p}}}{D_{\mathrm{p}}-1} D_{\min }\left[1-\left(\frac{D_{\min }}{D_{\max }}\right)^{D_{\mathrm{p}}-1}\right] \\
\tau_{\mathrm{av}}=\frac{1}{2}\left[1+\frac{1}{2 \sqrt{1-\varphi}}+\sqrt{1-\varphi} \frac{\sqrt{\left(\frac{1}{\sqrt{1-\varphi}}-1\right)^{2}+\frac{1}{4}}}{1-\sqrt{1-\varphi}}\right]
\end{gathered}
$$

\subsection{Fractal Permeability Model for Porous Media}

The flow of fluid in porous media is regarded as the flow in a curved capillary. The size distribution of the capillary channel satisfies the fractal distribution. According to the modified Hagen-Poiseulle equation, the flow rate $q$ of a fluid passing through a curved capillary can be expressed as [56,57]:

$$
q(D)=\frac{\pi}{128} \frac{\Delta p}{L_{t}(D)} \frac{D^{4}}{\mu}
$$

Equation (26) is obtained by considering the capillary tube as a circle. Since the size distribution of capillary channels satisfies the fractal distribution, the total flow rate $Q$ can be obtained by integrating the flow rate in a single root canal from the minimum pore diameter $D_{\min }$ to the maximum pore diameter $D_{\max }$ :

$$
Q=-\int_{D \min }^{D \max } q(D) d N(D)=\frac{\pi}{128 \mu} \frac{\Delta p}{L L_{0}^{D_{\mathrm{t}}-1}} \frac{D_{\mathrm{p}}}{3+D_{\mathrm{t}}-D_{\mathrm{p}}} D_{\max }^{3+D_{\mathrm{t}}}\left[1-\left(\frac{D_{\min }}{D_{\max }}\right)^{D_{\mathrm{p}}}\left(\frac{D_{\min }}{D_{\max }}\right)^{3+D_{\mathrm{t}}-2 D_{\mathrm{p}}}\right]
$$

Because $1<D_{\mathrm{t}}<2$ and $1<D_{\mathrm{p}}<2$, the exponent satisfies $3+D_{t}-2 D_{\mathrm{p}}>0$. And there is $0<\left(\frac{D_{\min }}{D_{\max }}\right)^{3+D_{\mathrm{t}}-2 D_{\mathrm{p}}}<1$, according to the criterion $\left(\frac{D_{\min }}{D_{\max }}\right)^{D_{\mathrm{p}}}<10^{-2}$ of Yu and Li [49], Equation (27) can be simplified into:

$$
Q=\frac{\pi}{128 \mu} \frac{\Delta p}{L L_{0}^{D_{\mathrm{t}}-1}} \frac{D_{\mathrm{p}}}{3+D_{\mathrm{t}}-D_{\mathrm{p}}} D_{\max }^{3+D_{\mathrm{t}}}
$$


According to Darcy's law, permeability can be expressed as:

$$
K=\frac{Q \mu L}{A \Delta p}
$$

where $A$ represents the cross section area of capillary bundle passing through the fluid. If the approximately equivalent capillary section is the flow section, and the surface porosity is assumed to be equal to the volume porosity. Then according to the fractal principle, the total pore area and the flow section area on the flow section can be expressed as [6]:

$$
\begin{gathered}
A_{\mathrm{p}}=-\int_{D_{\min }}^{D_{\max }} \frac{1}{4} \pi D^{2} \mathrm{~d} N=\int_{D_{\min }}^{D_{\max }} \frac{1}{4} \pi D^{2} D_{\mathrm{p}} D_{\max }^{D_{\mathrm{p}}} D^{-\left(D_{\mathrm{p}}+1\right)} \mathrm{d} D \\
=\frac{\pi D_{\mathrm{p}} D_{\max }^{2}}{4\left(2-D_{\mathrm{p}}\right)}\left[1-\left(\frac{D_{\min }}{D_{\max }}\right)^{2-D_{\mathrm{p}}}\right] \\
A=\frac{A_{\mathrm{p}}}{\varphi}=\frac{\pi D_{\mathrm{p}} D_{\max }^{2}}{4 \varphi\left(2-D_{\mathrm{p}}\right)}\left[1-\left(\frac{D_{\min }}{D_{\max }}\right)^{2-D_{\mathrm{p}}}\right]
\end{gathered}
$$

Because of $\varphi=\left(\frac{D_{\min }}{D_{\max }}\right)^{2-D_{\mathrm{p}}}$, the cross-section area can be simplified to [11]:

$$
A=\frac{\pi(1-\varphi) D_{\mathrm{p}} D_{\max }^{2}}{4 \varphi\left(2-D_{\mathrm{p}}\right)}
$$
and (32):

The permeability expression of pore media can be obtained by combining Equations (28), (29)

$$
K=\frac{1}{32} \frac{\varphi}{1-\varphi} \frac{1}{L_{0}^{D_{\mathrm{t}}-1}} \frac{2-D_{\mathrm{p}}}{3+D_{\mathrm{t}}-D_{\mathrm{p}}} D_{\max }^{1+D_{\mathrm{t}}}
$$

Substitute Equations (4) and (8) into Equation (33) to get the permeability expression of porous media composed of clusters of type I:

$$
K_{\mathrm{I}}=\frac{1}{128} \frac{\varphi}{1-\varphi}\left(\sqrt[3]{\frac{4 \pi}{3(1-\varphi)}}\right)^{1-D_{\mathrm{t}}}\left(4 \sqrt[3]{\frac{1}{9}} \sqrt[6]{\frac{1}{48}} \sqrt{\frac{\varphi}{\tau_{\mathrm{av}}}} \sqrt[3]{\frac{1}{1-\varphi}}\right)^{1+D_{\mathrm{p}}} \frac{2-D_{\mathrm{p}}}{3+D_{\mathrm{t}}-D_{\mathrm{p}}} D_{\mathrm{f}}^{2}
$$

By substituting Equations (12) and (14) into Equation (33), the relationship between porous media permeability formed by type II clusters and matrix particle size, porosity and fractal dimension can be obtained:

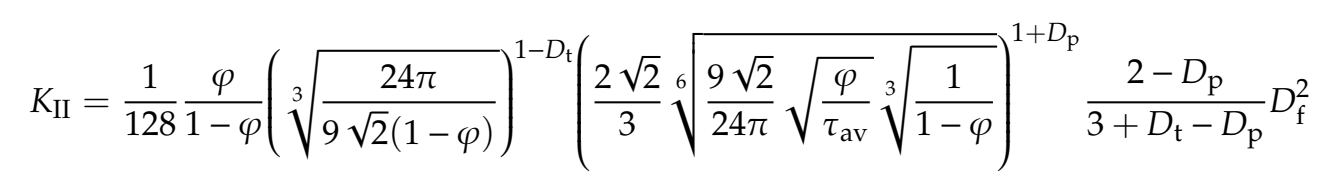

where $D_{\mathrm{f}}$ is the diameter of particles.

If Equation (25) is substituted into Equations (34) and (35), the permeability under the two ideal modes is a function of reservoir porosity, tortuosity fractal dimension, integral shape dimension of pore surface and radius of solid particles. The dimensionless permeability can be obtained by dividing Equations (34) and (35) by $D_{\mathrm{f}}^{2}$.

\section{Model Validation}

There have been many researches on porous media permeability model based on fractal theory, the most classic one is the $\mathrm{KC}$ equation, although this equation is strictly applicable to homogeneous media or actual random filled fiber media. Based on the fractal porous media pore space geometry 
hypothesis, Costa improved the KC equation and verified its validity [33]. The improved KC equation can be expressed as

$$
K=C_{\mathrm{kc}} \frac{\varphi^{n+1}}{(1-\varphi)^{n}}
$$

where $C_{\mathrm{kc}}$ and $n$ both are the empirical constant, which is related to particle shape and tortuosity. Although appropriate parameters can be set through experience, so that Equation (36) can better match experimental data, the significance of these parameters is not clear, which needs to be determined through experiments. Xiao et al., $\mathrm{Xu}$ and $\mathrm{Yu}$ continued to improve the classical KC equation based on the fractal bundle model $[25,28]$. The Xiao's permeability model can be expressed as:

$$
K=\left(\frac{4-D_{\mathrm{p}}}{D_{\mathrm{p}}}\right)^{1 / 2} \frac{\left[4\left(2-D_{\mathrm{p}}\right)\right]^{\left(1+D_{\mathrm{t}}\right) / 2}\left(\pi D_{\mathrm{p}}\right)^{\left(1-D_{\mathrm{t}}\right) / 2}}{128\left(3-D_{\mathrm{p}}+D_{\mathrm{t}}\right) \ln ^{2} \varphi}\left(\frac{\varphi}{1-\varphi}\right)^{\left(1+D_{\mathrm{t}}\right) / 2} D_{\mathrm{f}}^{2}
$$

According to Equations (36) and (37) and experimental data, we verified the model. As can be seen from Figure 5, the permeability of model I and model II are also different due to the different arrangement of particles, and the permeability of model I is relatively larger. In general, the permeability of our model increases with the increase of porosity, and the permeability changes rapidly when the porosity approaches 0 and 1 . In order to verify the proposed model accurately, we made artificial cores with an average porosity of $5 \%$ to $30 \%$ and measured the corresponding permeability. As shown in Figure 5, our experimental permeability is very close to the proposed model permeability when the porosity is between $5 \%$ and $30 \%$. In addition, we also compared the Costa's experimental data with the model we proposed [33]. The comparison results show that when the porosity is between $30 \%$ and $60 \%$, our model is closer to the experimental data of Costa than Xiao's model. Therefore, the comparison between our experimental data, Costa's experimental data and the proposed model shows that our model is more consistent with the experimental data of medium and low porosity $(5 \% \sim 60 \%)$, while Xiao's model is more consistent with the data of high porosity $(>60 \%)$ [28]. Therefore, our model is more suitable for predicting medium and low porosity porous media permeability. At the same time, the accuracy of the model is proved by comparing the results.

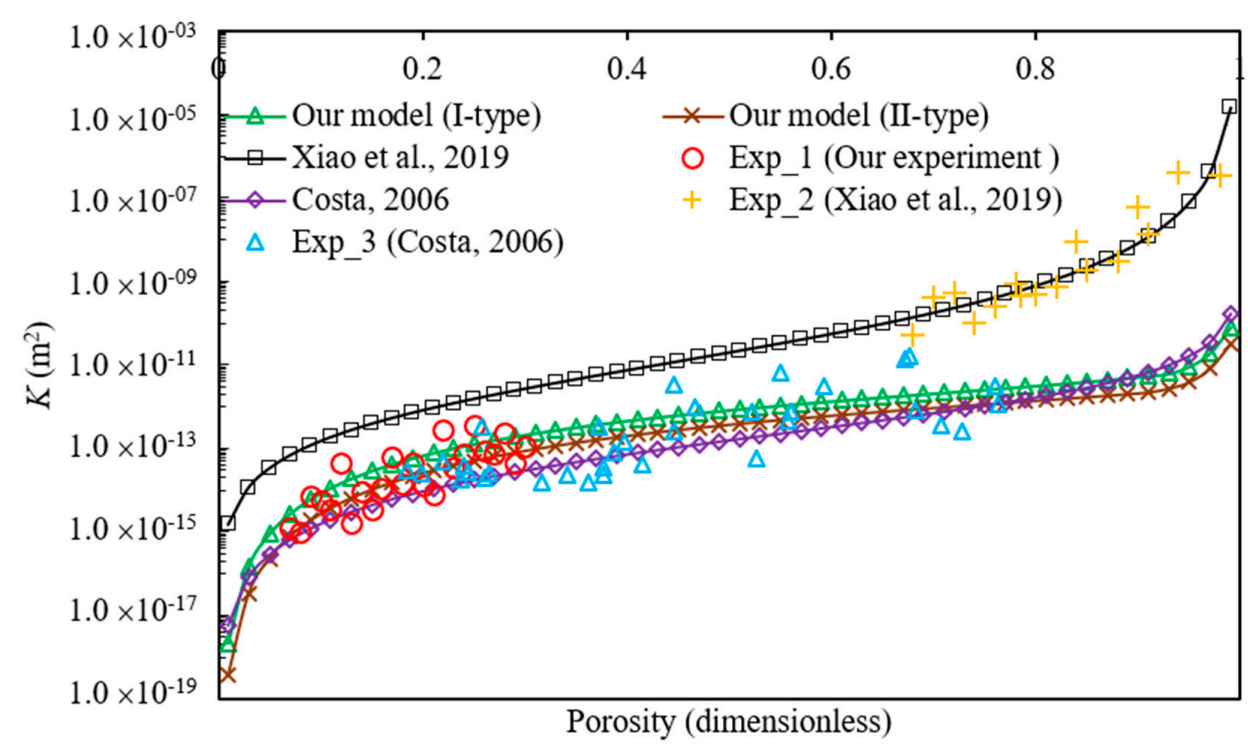

Figure 5. A comparison between the absolute permeability of porous media by the proposed fractal model and existing experimental data (Best parameters are $C_{\mathrm{kc}}=1.77 \times 10^{-12} \mathrm{~m}^{2} ; n=1.07$; $D_{\mathrm{f}}=6 \times 10^{-5} \mathrm{~m}$ ). 


\section{Results Discussion and Analysis}

Figure 6 shows the relationship between porosity and pore diameter of the largest bundle. It can be seen from Figure 6 that the equivalent capillary bundle pore diameter increases with the increase of porosity. And with the increase of porosity, the diameter difference between the two modes becomes larger and larger. Due to the different arrangement of particles, the equivalent pore diameter is not the same.

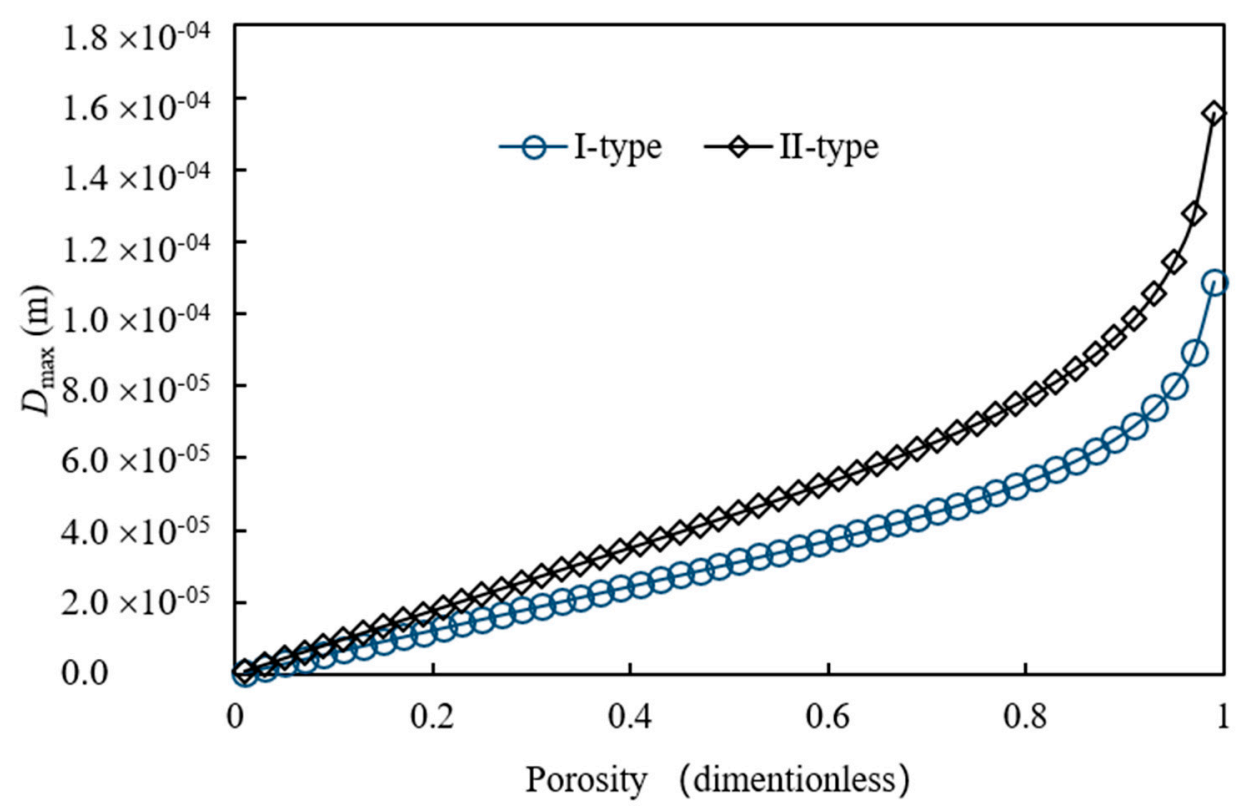

Figure 6. The relationship between pore diameter and porosity of capillary bundle model section.

By substituting Equations (4), (8), (24), and (25) into Equations (22) and (23), it can be known that the tortuosity fractal dimension and the pore area fractal dimension are all functions of porosity. Figure 7 shows the effect of porosity on fractal dimension. With the increase of porosity, the tortuosity fractal dimension decreases, which means that the greater the porosity, the more bent the capillary bundle is, and the longer the capillary bundle is. With the increase of porosity, the pore area fractal dimension also increases, and the maximum pore area on the cross section also increases. In addition, the arrangement of solid particles has little influence on fractal dimension. The pore area fractal dimension and tortuous fractal dimension of type I arrangement are larger than those of type II arrangement under different arrangement modes of solid particles.

Figures 8 and 9 respectively show the influence of the tortuosity fractal dimension on permeability of porous media and the influence of pore area fractal dimension on permeability of porous media. The permeability of porous media has a strong nonlinear relationship with the tortuosity fractal dimension and the integral shape dimension of pore surface. Figure 8 shows that the permeability decreases with the increase of tortuosity fractal dimension, and the permeability changes rapidly when the tortuosity is close to 1 . Figure 9 shows that the permeability of porous media increases with the increase of the pore area fractal dimension, and the permeability changes faster when the pore area fractal dimension approaches 2. By comparing the two figures, the tortuosity fractal dimension has the opposite effect on permeability with the pore area fractal dimension. It can also be seen that the larger the radius of particles, the greater the permeability. 


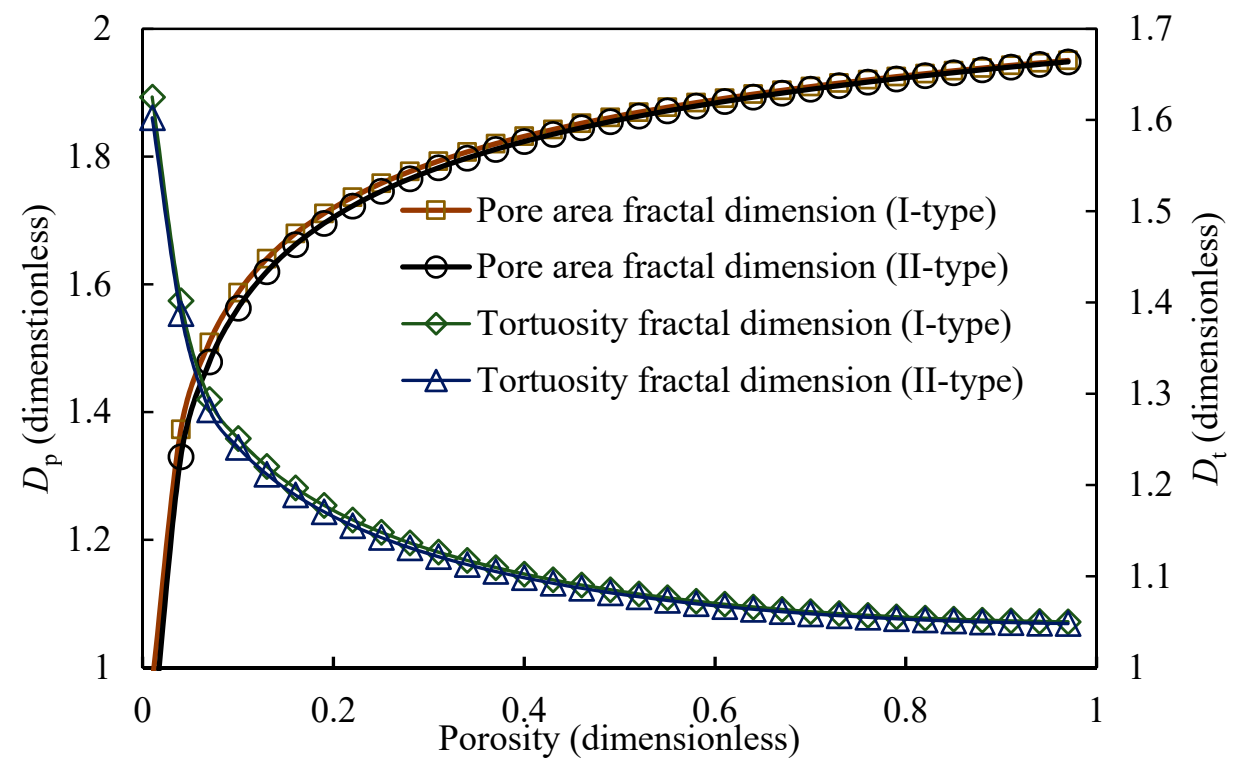

Figure 7. Influence of porosity on the tortuosity fractal dimension $\left(D_{\mathrm{t}}\right)$ and pore area fractal dimension $\left(D_{\mathrm{p}}\right)$ in reservoir rock porous media.

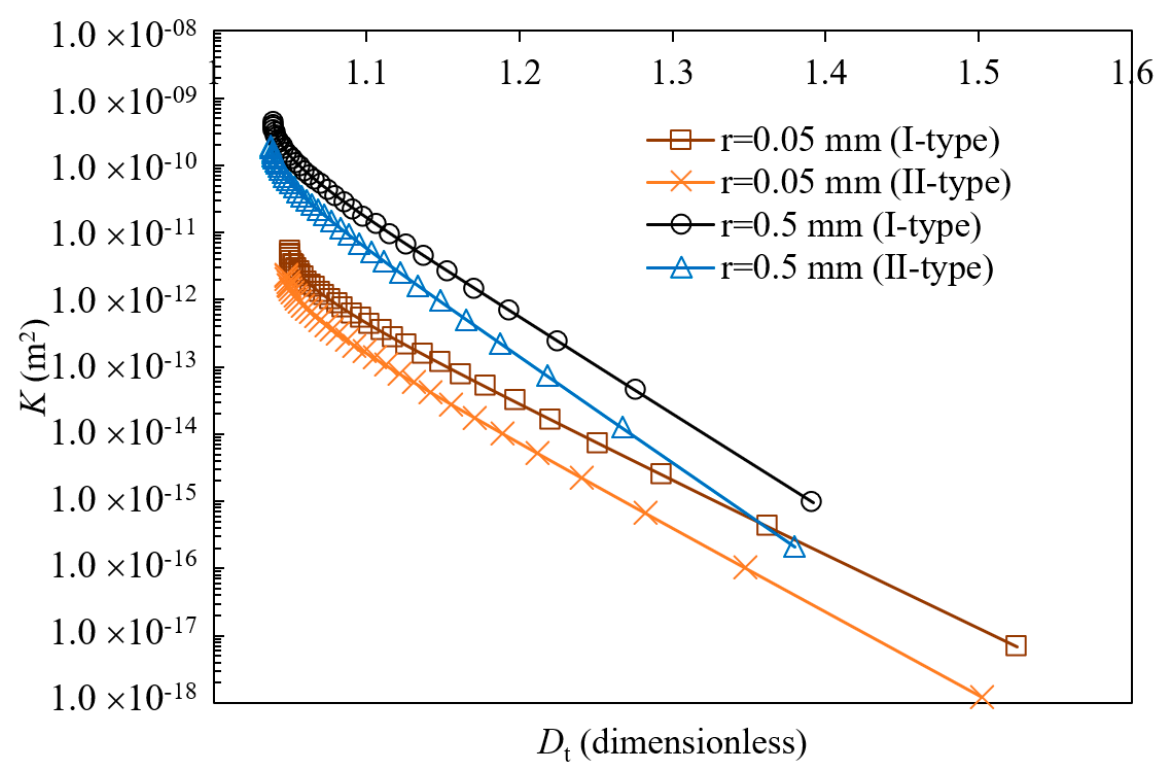

Figure 8. Effect of tortuosity fractal dimension on permeability of porous media. 


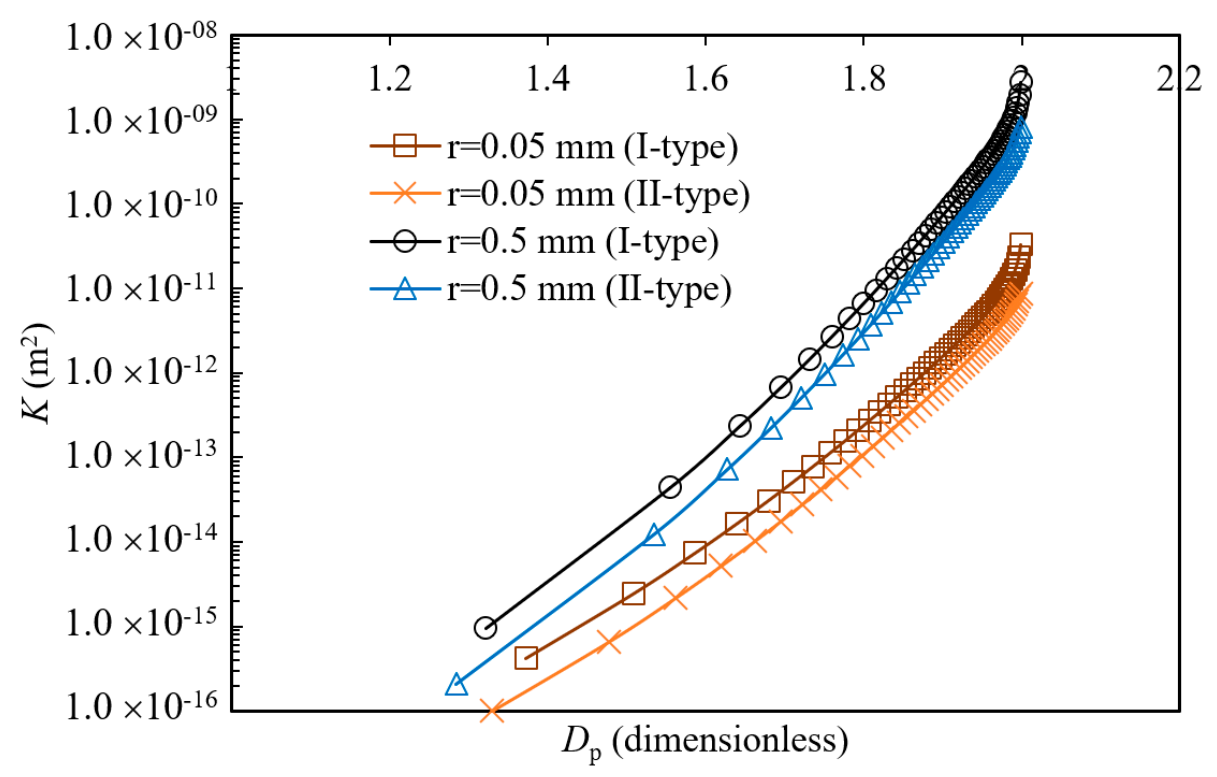

Figure 9. Effect of pore area fractal dimension on permeability of porous media.

In order to analyze the influence of particle arrangement mode on permeability, the permeability difference coefficient $R$ caused by different particle arrangement mode is introduced. The connotation of $R$ is to reflect the influence degree of solid particle arrangement mode on permeability. The larger $R$ value is, the greater the influence degree of permeability is by solid particle arrangement mode. $R$ can be expressed as:

$$
R=\frac{K_{\mathrm{I}}-K_{\mathrm{II}}}{K_{\mathrm{II}}}=\left(\sqrt[3]{\frac{3 \sqrt{2}}{6}}\right)^{1-D_{\mathrm{t}}}\left(3 \sqrt{2} \sqrt[3]{\frac{1}{9}} \sqrt[6]{\frac{\sqrt{2} \pi}{36}}\right)^{1+D_{\mathrm{p}}}-1
$$

Figure 10 shows the effect of changes in porosity and solid particle radius on the difference coefficient. As can be seen from the figure, under the same particle radius, with the change of porosity, the difference coefficient is larger when the porosity is close to 0 and 1 , and smallest when the porosity is close to 0.6 . Therefore, the prediction of permeability of porous media with high porosity and low porosity should pay particular attention to the influence of particle arrangement. In addition, the difference coefficient increases with the increase of the radius of solid particles. This indicates that the arrangement of particles has a great influence on the permeability of porous media when the particle radius is large. It can be seen from Equation (38) that the difference coefficient is a function of the pore area fractal dimension and the tortuosity fractal dimension. Figure 11 shows the relationship between fractal dimension and permeability difference coefficient. The permeability difference coefficient decreases first and then increases with the increase of pore area fractal dimension, reaching the minimum when its value is close to 2 . At the same time, as the tortuosity fractal dimension increases, the permeability difference coefficient decreases first and then increases, reaching the minimum when its value is close to 1 . The permeability difference coefficient changes rapidly when the pore area fractal dimension approaches 2 and the tortuosity fractal dimension approaches 1 , respectively. 


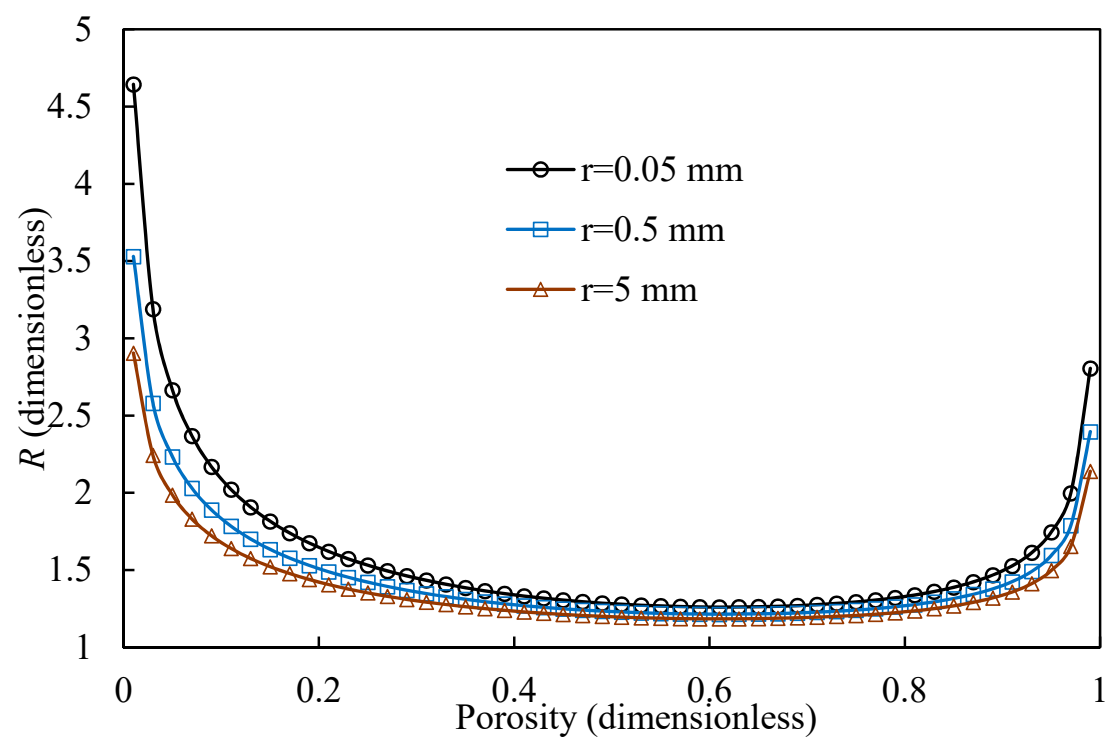

Figure 10. The relationship between porosity and permeability differential coefficient.

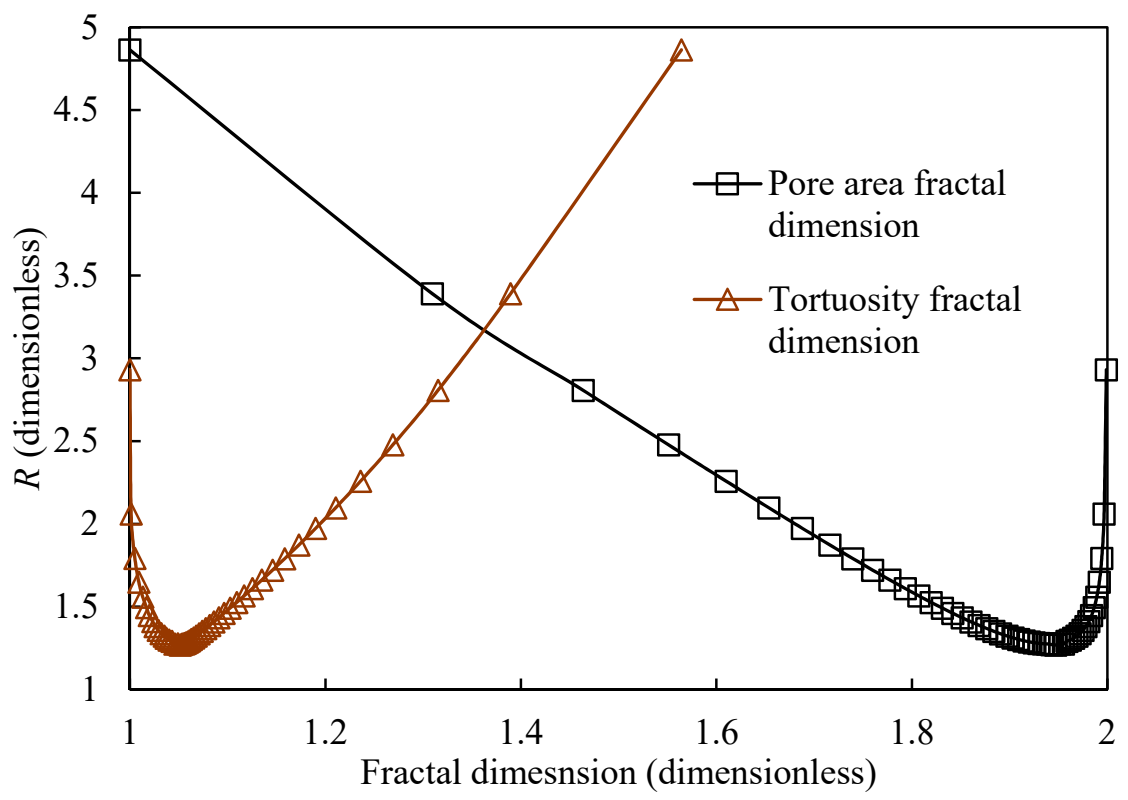

Figure 11. The relationship between the fractal dimension and the permeability difference coefficient $(R)$.

\section{Conclusions}

In this paper, we derive a novel porous media permeability model considering irregular pore space based on fractal theory and ideal particle space geometry. The results show that our model is more suitable for medium and low porosity porous media. In addition, the following conclusions are obtained through multivariate analysis: (1) the equivalent capillary bundle pore diameter increases with the increase of porosity; (2) tortuosity fractal dimension has a negative correlation with porosity, while the pore area fractal dimension of the pore surface has a positive correlation with porosity; (3) the permeability is negatively correlated with the tortuosity fractal dimension and positively correlated with the pore area fractal dimension and particle radius. When the tortuosity fractal dimension is close to 1 and the pore area fractal dimension is close to 2 , the faster the permeability changes, the greater the impact. (4) different particle arrangement has great influence on porous media permeability. When the porosity is close to 0 and close to 1 , the permeability is especially affected by the greater the difference coefficient. In addition, the larger the particle radius is, the greater the permeability 
difference coefficient is, and the greater the permeability difference is for different particle arrangement. (5) With the increase of fractal dimension, the permeability difference coefficient first decreases and then increases. When the pore area fractal dimension approaches 2 , the permeability difference coefficient changes quickly and reaches the minimum value, and when the tortuosity fractal dimension approaches 1 , the permeability difference coefficient changes quickly and reaches the minimum value.

Author Contributions: Conceptualization, Y.H. and J.Z.; methodology, Q.W.; formal analysis, S.X.; investigation, Q.W.; writing—original draft preparation, Q.W.; writing—review and editing, Y.H. and J.Z.; supervision, H.J. All authors have read and agreed to the published version of the manuscript.

Funding: This research was funded by National Natural Science Foundation of China grant number 51404204 And the APC was funded by National Science and Technology Major Project of the Ministry of Science and Technology of China grant number 2016ZX05060. The authors would like to acknowledge the financial support of the China Scholarship Council.

Conflicts of Interest: The authors declare no conflict of interest.

\section{References}

1. Mousavi, M.; Prodanovic, M.; Jacobi, D. New classification of carbonate rocks for process-based pore-scale modeling. SPE J. 2013, 18, 243-263. [CrossRef]

2. Wu, K.; Chen, Z.X.; Li, X.F. Real gas transport through nanopores of varying cross-section type and shape in shale gas reservoirs. Chem. Eng. J. 2015, 281, 813-825. [CrossRef]

3. Wu, K.; Chen, Z.X.; Li, X.F.; Guo, C.; Wei, M. A model for multiple transport mechanisms through nanopores of shale gas reservoirs with real gas effect-adsorption-mechanic coupling. Int. J. Heat Mass Transf. 2016, 93, 408-426. [CrossRef]

4. Hosa, A.; Curtis, A.; Wood, R. Calibrating Lattice Boltzmann flow simulations and estimating uncertainty in the permeability of complex porous media. Adv. Water Resour. 2016, 94, 60-74. [CrossRef]

5. Chen, Z.L.; Wang, N.T.; Sun, L.; Tan, X.H.; Deng, S. Prediction method for permeability of porous media with tortuosity effect based on an intermingled fractal units model. Int. J. Eng. Sci. 2017, 121, 83-90. [CrossRef]

6. Pitchumani, R.; Ramakrishnan, B. A fractal geometry model for evaluating permeabilities of porous preforms used in liquid composite molding. Int. J. Heat Mass Transf. 1999, 42, 2219-2232. [CrossRef]

7. Miguel, A.F.; Rosa, R.; Silva, A.M. Fractal geometry description of the permeability of a natural fissured rock. In Proceedings of the 9th International Congress on Deterioration and Conservation of Stone; Elsevier Science B.V.: Amsterdam, The Netherlands, 2000; Volume 1, pp. 595-600.

8. Karacan, Q.; Halleck, P.M. A Fractal Model for Predicting Permeability around Perforation Tunnels Using Size Distribution of Fragmented Grains. J. Pet. Sci. Eng. 2003, 40, 159-176. [CrossRef]

9. Dravis, J.J. Carbonate applied to hydrocarbon exploration and exploitation, HTC Intetnal report. Training course note. 2007. Available online: www.dravisinterests.com (accessed on 7 November 2009).

10. Lucia, F.L. Petrophysical Parameters Estimated from Visual Descriptions of Carbonate Rocks: A Field Classification of Carbonate Pore Space. In Proceedings of the 1981 SPE Annual Technical Conference and Exhibition, San Antonio, TX, USA, 5-7 October 1983.

11. Wu, J.S. Analysis of Resistance for Flow Through Porous Media; Zhejiang University: Hangzhou, China, 2006.

12. Gebart, B.R. Permeability of unidirectional reinforcements for RTM. J. Compos. Mater. 1992, 26, 1100-1133. [CrossRef]

13. Othman, M.R.; Helwani, Z.; Martunus. Simulated fractal permeability for porous membranes. Appl. Math. Model. 2010, 34, 2452-2464. [CrossRef]

14. Zhu, F.; Hu, W.X.; Cao, J.; Sun, F.; Liu, Y.; Sun, Z. Micro/nanoscale pore structure and fractal characteristics of tight gas sandstone: A case study from the Yuanba area, northeast Sichuan Basin, China. Mar. Pet. Geol. 2018, 98, 116-132. [CrossRef]

15. Qin, X.; Cai, J.C.; Xu, P. A fractal model of effective thermal conductivity for porous media with various liquid saturation. Int. J. Heat Mass Transf. 2019, 128, 1149-1156. [CrossRef]

16. Wang, Q.; Hu, Y.; Zhao, J.; Ren, L.; Zhao, C.; Zhao, J. Multiscale Apparent Permeability Model of Shale Nanopores Based on Fractal Theory. Energies 2019, 12, 3381. [CrossRef]

17. Zhao, J.; Wang, Q.; Hu, Y.; Ren, L.; Zhao, C. Numerical investigation of shut-in time on stress evolution and tight oil production. J. Pet. Sci. Eng. 2019, 179, 716-733. [CrossRef] 
18. Maik, N.K.; Sirisha, M.; Inani, A. Permeability characterization of polymer matrix composites by RTM/VARTM. Prog. Aerosp. Sci. 2014, 65, 22-40.

19. Parnas, R.S.; Salem, A.J.; Sadiq, T.A.; Wang, H.P.; Advani, S.G. The interaction between micro-and macro-scopic flow in RTM preforms. Compos. Strcut. 1994, 27, 83-107. [CrossRef]

20. Kozeny, J. Ueber kapillare leitung des wassers im boden. Sitzh. Akad. Wiss. Wein 1927, 136, 271-306.

21. Carman, P.C. Fluid flow through granular beds. Trans. Inst. Chem. Eng. 1937, 15, 150-167. [CrossRef]

22. Griffin, P.R.; Grove, S.M.; Russell, P.; Short, D.; Summerscales, J.; Guild, F.J.; Taylor, E. The effect of reinforcement architecture on the long-range flow in fibrous reinforcements. Compos. Manuf. 1995, 6, 221-235. [CrossRef]

23. Khabbazi, A.E. Numerical and Analytical Characterization of Transport Properties for Single Phase Flows in Granular Porous Media; University of Toronto: Toronto, ON, Canada, 2015.

24. Yu, B.M.; Lee, L.J.; Cao, H.Q. Fractal characters of pore microstructures of textile fabrics. Fractals 2001, 9, 155-163. [CrossRef]

25. Xu, P.; Yu, B.M. Development a new form of permeability and Kozeny-Carman constant for homogeneous porous media by means of fractal geometry. Adv. Water Resour. 2008, 31, 74-81. [CrossRef]

26. Xiao, B.Q.; Fan, J.T.; Ding, F. A fractal analytical model for the permeabilities of fibrous gas diffusion layer in proton exchange membrane fuel cells. Electrochem. Acta 2014, 134, 222-231. [CrossRef]

27. Xiao, B.Q.; Wang, W.; Zhang, X.; Long, G.; Fan, J.T.; Chen, H.; Deng, L. A novel fractal solution for permeability and Kozeny-Carman constant of fibrous porous media made up of solid particles and porous fibers. Powder Technol. 2019, 349, 92-98. [CrossRef]

28. Xiao, B.Q.; Fan, J.T.; Ding, F. A novel fractal model for relative permeability of gas diffusion layer in proton exchange membrane fuel cell with capillary pressure effect. Fractals 2019, 27, 1950012. [CrossRef]

29. Shou, D.H.; Fan, J.; Ding, F. A difference-fractal model for the permeability of fibrous porous media. Phys. Lett. A 2010, 374, 1201-1204. [CrossRef]

30. Zhang, L.Z. A fractal model for gas permeation through porous membranes. Int. J. Heat Mass Transf. 2012, 55, 1716-1723. [CrossRef]

31. Zhu, Q.Y.; Xie, M.H.; Yang, J.; Li, Y. A fractal model for the coupled heat and mass transfer in porous fibrous media. Int. J. Heat Mass Transf. 2011, 54, 1400-1409. [CrossRef]

32. Zhu, Q.Y.; Xie, M.H.; Yang, J.; Li, Y. Analytical determination of permeability of porous fibrous media with consideration of electro kinetic phenomena. Int. J. Heat Mass Transf. 2012, 9, 365-372.

33. Costa, A. Permeability-porosity relationship: A reexamination of the Kozeny-Carman equation based on a fractal pore-space geometry assumption. Geophys. Res. Lett. 2005, 33, L02318. [CrossRef]

34. Cihan, A.; Sukop, M.C.; Tyner, J.S.; Perfect, E.; Huang, H. Analytical predictions and lattice Boltzmann simulations of intrinsic permeability for mass fractal porous media. Vadose Zone J. 2009, 8, 187-196. [CrossRef]

35. Garrison, J.R.; Pearn, W.C.; Vonrosenberg, D.U. The fractal menger sponge and Sierpinski carpet as models for reservoir rock/por systems: I. theory and image analysis of Sierpinski carpets. In Situ 1992, 16, 351-406.

36. Garza-Lopez, R.A.; Naya, L.; Kozak, J.J. Tortuosity factor for permeant flow through a fractal solid. J. Chem. Phys. 2000, 112, 9956-9960. [CrossRef]

37. Cihan, A.; Perfect, E.; Tyner, J.S. Water retention models for scale-variant and scale-invariant drainage of mass prefractal porous media. Vadose Zone J. 2007, 6, 786-792. [CrossRef]

38. Atzeni, C.; Pia, G.; Sanna, U.; Spanu, N. A fractal model of the porous microstructure of earth-based materials. Constr. Build. Mater. 2008, 22, 1607-1613. [CrossRef]

39. Pia, G.; Sanna, U. An intermingled fractal units model and method to predict permeability in porous rock. Int. J. Eng. Sci. 2014, 75, 31-39. [CrossRef]

40. Pia, G.; Sanna, U. An intermingled fractal units model to evaluate pore size distribution influence on thermal conductivity values in porous materials. Appl. Therm. Eng. 2014, 65, 330-336. [CrossRef]

41. Pia, G.; Sanna, U. Intermingled fractal units model and electrical equivalence fractal approach for prediction of thermal conductivity of porous materials. Appl. Therm. Eng. 2013, 61, 186-192. [CrossRef]

42. Turcio, M.; Reyes, J.; Camacho, R.; Lira-Galeana, C.; Vargas, R.O.; Manero, O. Calculation of effective permeability for the BMP model in fractal porous media. J. Pet. Sci. Eng. 2013, 103, 51-60. [CrossRef]

43. Bautista, F.; Santos, J.M.; Puig, J.E.; Manero, O. Understanding thixotropic and antithixotropic behavior of viscoelastc micellar solutions and liquid crystalline dispersions. I. the model. J. Non-Newton. Fluid Mech. 1999, 80, 93-113. [CrossRef] 
44. Miao, T.J.; Chen, A.; Zhang, L.W. A novel fractal model for permeability of damaged tree-like branching networks. Int. J. Heat Mass Transf. 2018, 127, 278-285. [CrossRef]

45. Gostick, J.T.; Fowler, M.W.; Loannidis, M.A.; Pritzker, M.D.; Volfkovich, Y.M.; Sakars, A. Capillary pressure and hydrophilic porosity in gas diffusion layers for polymer electrolyte fuel cells. J. Power Sources 2006, 156, 375-387. [CrossRef]

46. Shou, D.H.; Fan, J.; Ding, F. Hyfraulic permeability of fibrous porous media. Int. J. Heat Mass Transf. 2011, 54, 4009-4018. [CrossRef]

47. Ju, Y.; Xi, C.; Zhang, Y.; Mao, L.T.; Gao, F.; Xie, H.P. Laboratory In Situ CT Observation of the Evolution of 3D Fracture Networks in Coal Subjected to Confining Pressures and Axial Compressive Loads: A Novel Approach. Rock Mech. Rock Eng. 2018, 51, 3361-3375. [CrossRef]

48. Fan, W.P.; Sun, H.; Yao, J.; Fan, D.Y.; Zhang, K. A new fractal transport model of shale gas reservoirs considering multiple gas transport mechanisms, multi-scale and heterogeneity. Fractals 2018, 26, 1850096. [CrossRef]

49. Yu, B.M.; Li, J.H. Some fractal characters of porous media. Fractals 2001, 9, 365-372. [CrossRef]

50. Yun, M.J.; Yu, B.M.; Zhang, B.; Huang, M.T. A geometry model for tortuosity of stream tubes in porous media with spherical particles. Chin. Phys. Lett. 2005, 22, 1464-1467.

51. Yun, M.J.; Yu, B.M.; Xu, P.; Wu, J.S. Geometrical models for tortuosity of streamlines in three-dimensional porous media. Can. J. Chem. Eng. 2006, 84, 301-309. [CrossRef]

52. Kou, J.L.; Tang, X.M.; Zhang, H.Y.; Lu, H.J.; Wu, F.; Xu, Y.; Dong, Y. Tortuosity for streamlines in porous media. Chin. Phys. Rev. 2012, 21, 044701. [CrossRef]

53. Daigle, H. Application of critical path analysis for permeability prediction in natural porous media. Adv. Water Resour. 2016, 96, 43-54. [CrossRef]

54. Wheatcraft, S.W.; Tyler, S.W. An explanation of porous dispersivity in heterogeneous aquifers using concepts of fractal geometry. Water Resour. Res. 1988, 24, 566-578. [CrossRef]

55. Sun, Z.; Shi, J.T.; Wu, L.; Zhang, T.; Feng, D.; Huang, L.; Shi, Y.; Ramachandean, H.; Li, X. An analytical model for gas transport through elliptical nanopores. Chem. Eng. Sci. 2019, 199, 199-209. [CrossRef]

56. Cussler, E.L. Diffusion-Mass Transfer in Fluid Systems; Cambridge University Press: Cambridge, UK, 2000.

57. Skjeltorp, A.T.; Feder, A.A. (Eds.) Fractals in Physics; Elsevier: Amsterdam, The Netherlands, 1990.

(C) 2020 by the authors. Licensee MDPI, Basel, Switzerland. This article is an open access article distributed under the terms and conditions of the Creative Commons Attribution (CC BY) license (http://creativecommons.org/licenses/by/4.0/). 